\title{
The robust Merton problem of an ambiguity averse investor
}

\author{
Sara Biagini $^{1}$ (D) Mustafa Ç. Pınar ${ }^{2}$
}

Received: 9 February 2016 / Accepted: 11 March 2016 / Published online: 26 March 2016

(C) Springer-Verlag Berlin Heidelberg 2016

\begin{abstract}
We derive a closed form portfolio optimization rule for an investor who is diffident about mean return and volatility estimates, and has a CRRA utility. Confidence is here represented using ellipsoidal uncertainty sets for the drift, given a (compact valued) volatility realization. This specification affords a simple and concise analysis, as the agent becomes observationally equivalent to one with constant, worst case parameters. The result is based on a max-min Hamilton-Jacobi-Bellman-Isaacs PDE, which extends the classical Merton problem and reverts to it for an ambiguity-neutral investor.
\end{abstract}

Keywords Robust optimization · Merton problem · Volatility uncertainty · Ellipsoidal uncertainty on mean returns · Hamilton-Jacobi-Bellman-Isaacs equation

Mathematics Subject Classification $91 \mathrm{G} 10 \cdot 91 \mathrm{~B} 25 \cdot 90 \mathrm{C} 25 \cdot 90 \mathrm{C} 46 \cdot 90 \mathrm{C} 47$

JEL Classification G11 · C61

\section{Introduction}

Traditionally, financial modelling heavily relies on the choice of an underlying probability measure $P$, which is chosen to incorporate the statistical and stochastic nature of market price movements. As early back as the works of Bachelier, Samuelson and Black, Scholes and Merton, the underlying risk factors — such as stock prices or interest rates-have been modeled as Markovian diffusions (with possible jumps) under $P$. However, as has become

Sara Biagini

sbiagini@luiss.it

Mustafa Ç. Pınar

mustafap@bilkent.edu.tr

1 Department of Economics and Finance, LUISS G. Carli, 00197 Rome, Italy

2 Department of Industrial Engineering, Bilkent University, 06800 Bilkent, Ankara, Turkey 
quite agreed upon, the complexity of the global economic and financial dynamics renders impossible the precise identification of the probability law of the evolution of the risk factors. Unavoidably, financial modelling is inherently subject to model ambiguity, which also appears under the appellation of Knightian uncertainty.

In the presence of model ambiguity, one may admit various degrees of severity. One may deal with model misspecification only at the level of the equivalence class of $P$, or go beyond and take into account a family of non dominated models. The core issue of portfolio optimization has been widely investigated over the last twenty years in the multiple priors context. The investor has a pessimistic view of the odds, and takes a max-min (also known as robust) approach to the problem, first minimizing a utility functional over the priors and afterwards maximizing over the investment strategies. The pessimistic behavior has been thoroughly axiomatized via the so-called ambiguity averse preferences, see Maccheroni et al. [16,17] for recent developments.

In the non-dominated case, we are aware of only a few papers, notably: HernàndezHernàndez and Schied [11], where uncertainty in the volatility is due to an unobservable factor; Nutz [21] in discrete time; Lim et al. [15] work in a jump-diffusion context, with ambiguity on drift, volatility and jump intensity, and provide results of deep intuition for utility maximization from terminal wealth, but the mathematics in our opinion are not fully worked out. The closest to our setup is probably the inspiring Lin and Riedel [25]. Here the authors work in a diffusion context, and uncertainty is modelled by allowing drift and volatility to vary in two constant order intervals. The optimization is then performed via a robust control (G-Brownian motion) technique, thus the uncertain volatility matrix is restricted to be of diagonal type.

After the first version of the present work appeared, Neufeld and Nutz [20] posted on the Arxiv a very nice preprint, where, in a continuous time setting, the agent maximizes CRRA expected utility of terminal wealth with duality methods. Remarkably, the stock prices are allowed to be general discontinuous semimartingales and uncertainty is modelled by allowing the predictable characteristics triplet to be valued in a general convex set, with compact projection on the first two characteristics. The strategies are however required to be compact in order to apply a suitable version of the Sion's minimax theorem.

On the contrary, in the dominated priors case there is a rich literature. We content ourselves with citing Chen and Epstein [4], Garlappi et alii [13], Maenhout [18], Föllmer et alii [12] for a comprehensive review and references, and, more recently, the work by Owari [22].

In such an active environment, the present paper offers a resolution of the robust non-dominated consumption/investment Merton problem, which is both simple and mathematically rigorous. We assume that the asset prices process is an $n$-dimensional diffusion, with a driving $n$-dimensional Wiener process. The investor is diffident about the constant drift and volatility estimates $\hat{\mu}$ and $\hat{\sigma}$. Thus, she considers as plausible all the (instantaneous) covariance matrices lying in a given compact set $K$. The compact $K$ is fairly general, need not be convex or verify some particular correlation constraint; the only requirement is that it satisfies a uniform non degeneracy condition. For a given realization of the process $\sigma$, the agent then considers all the drifts which take values in an ellipsoid centered at $\hat{\mu}$ :

$$
U_{\epsilon}(\Sigma)=\left\{u \in \mathbb{R}^{n} \mid(u-\hat{\mu})^{\prime} \Sigma^{-1}(u-\hat{\mu}) \leq \epsilon^{2}\right\},
$$

in which $\epsilon \geq 0$ is the radius of ambiguity, ' denotes the transpose operation and $\Sigma=\sigma \sigma^{\prime}$ is the variance-covariance matrix.

The problem of worst-case ( $\max -\mathrm{min}$ ) robust portfolio choice is a well-studied problem (see e.g., [5, 6, 8-10,13,14,23,24] for robust portfolio optimization in single period problems) under different representations of ambiguity. As observed in Sect. 2, Remark 3, part of our results holds for any uncertainty set for $(\Sigma, \mu)$ which is compact and non degenerate in $\Sigma$. 
Nevertheless, we choose the ellipsoidal representation for the ambiguous drifts for two main reasons. First, it is well- established in the Finance literature, see e.g. [13] and [14] for the robust mean-variance optimization. As Fabozzi et al. [8] say: "The coefficient realizations are assumed to be close to the forecasts, but they may deviate. They are more likely to deviate from their (instantaneous) means if their variability (measured by their standard deviation) is higher, so deviations from the mean are scaled by the inverse of the covariance matrix of the uncertain coefficients. The parameter $\epsilon$ corresponds to the overall amount of scaled deviations of the realized returns from the forecasts against which the investor would like to be protected."

Secondly, the non-linear but simple geometry of ellipsoids offers robustness that avoids a worst case which is a corner solution. In fact, one always gets a corner solution in a polyhedral hyper-rectangle or box representation, as in [25] where the drift (as well as volatility) is allowed to vary in an order interval $[\underline{\mu}, \bar{\mu}]$ (and $[\underline{\sigma}, \bar{\sigma}]$ ). In the dominated setup, the assumption of $k$-ignorance in [4] also amounts to a box ambiguity for the drift.

On a general level, an appealing feature of taking model uncertainty into account is that it offers a theoretical solution to the equity premium puzzle. As noted by Mehra and Prescott [19], the high levels of historical equity premium and the simultaneous moderate equity demand seem to be implied by unreasonable levels of risk aversion. Their conclusion was skeptical on the ability of a frictionless Arrow-Debreu economy to account for such empirical evidence. However, the works by Abel [1] and Cecchetti, Lam and Mark [3] addressed the equity premium puzzle by relaxing the hypothesis that the investor perfectly knows the probability law. The key point is that, in the multiple priors setup, the optimal equity demand depends on two aversion components: risk and ambiguity aversion. In accordance to these results, and the subsequent [4], [18] and [25], we find that robustness of decisions lowers the optimal demand of equity since the ambiguity and risk averse investor effectively behaves like a risk averse investor with an increased risk aversion coefficient (see Theorem 4 and the subsequent comments).

The paper is organized as follows. Section 2 contains the model specifications in the non dominated case and the consumption/investment problem formulation. The robust problem can be alternatively seen as a zero sum, two-players game between the agent and the adverse market (or the malevolent Nature). Theorem 1 provides a suitable version of the Martingale Principle, from which a sup-inf PDE of the Hamilton-Jacobi-Bellman-Isaacs type arises. Such equations are classical in game theory (see [7] for more information). In Proposition 1 , we show that the supremum and the infimum in this PDE are attained for any regular, monotone increasing and strictly concave function.

In order to exhibit an explicit solution, in Sect. 3 we focus on the case of a representative investor with CRRA utility. Given our uncertainty set specification, and for the next usage in Sect. 4, we freeze the volatility, to wit assume that it is constant and that ambiguity is present only in the drift. This is an interesting case per se, since the drift is subject to imprecision in estimations to a much greater extent than volatility. Then, we solve and provide the explicit solutions to the these partial problems, together with a saddle point analysis both for the infinite and finite horizon planning (Theorems 2, 3 and Sect. 3.2). In Sect. 4 we analyze the genuinely non dominated setup and prove our main result, Theorem 4 . In here, we find explicit solutions to the general robust problem and we provide a saddle point analysis of this agent-adverse market game. The findings are in line with the extant literature, in that the investor is observationally equivalent to one who has distorted, worst case, beliefs on drift and volatility (Eq. (22)). We conclude with some examples. 


\section{The general Merton problem under ambiguity aversion}

Consider the problem of an agent investing in $n$ risky assets and a riskless asset. Specifically, we work under the Black-Scholes-Merton market model assumptions. Namely, the riskless rate $r$ is constant and the risky assets dynamics are, for each $i=1, \ldots, n$ :

$$
d S_{t}^{i}=S_{t}^{i}\left(\mu^{i} d t+\sum_{j=1}^{n} \sigma^{i j} d W_{t}^{j}\right)
$$

where $\sigma^{i j}$ and $\mu^{i}$ are constants and $W$ is a standard, $n$-dimensional Brownian motion on a filtered space $\left(\Omega,\left(\mathcal{F}_{t}\right)_{t \geq 0}, P\right)$. In matrix-vector form, the above equation becomes:

$$
d S_{t}=\operatorname{Diag}\left(S_{t}\right)\left(\mu d t+\sigma d W_{t}\right),
$$

where $\operatorname{Diag}\left(S_{t}\right)$ denotes the diagonal $n \times n$ matrix with $i$-th diagonal element equal to $S_{t}^{i}$, $\mu$ is an $n$-vector and $\sigma$ is a $n \times n$ matrix. In addition, $\sigma$ is required to be invertible, so that the instantaneous covariance matrix $\Sigma=\sigma \sigma^{\prime}$ is also invertible.

Given the initial endowment $x>0$, the investor is allowed to trade and consume in a self-financing way. To be explicit, let $h=\left(h_{t}\right)_{t}$ denote the $n$-dimensional progressively measurable process, representing the number of shares of each asset held in portfolio, and let the progressively measurable, non-negative, scalar process $c$ indicate the consumption stream. Assume also that $\int_{0}^{\cdot} h_{s}^{\prime} \Sigma h_{s} d s$ and $\int_{0}^{\cdot} c_{s} d s$ are finite $P$-a.s. The wealth process $X$ is governed by the following stochastic differential equation:

$$
d X_{t}=\left(r X_{t}+h_{t}^{\prime} \operatorname{Diag}\left(S_{t}\right)(\mu-r \mathbf{1})-c_{t}\right) d t+h_{t}^{\prime} \operatorname{Diag}\left(S_{t}\right) \sigma d W_{t}
$$

in which $\mathbf{1}$ is the $n$-vector with all components equal to one. It is convenient to recast the wealth equation by the vector process $\theta$ of cash value allocated in each risky asset, i.e. $\theta_{t}:=\operatorname{Diag}\left(S_{t}\right) h_{t}$. Thus,

$$
d X_{t}=\left(r X_{t}+\theta_{t}^{\prime}(\mu-r \mathbf{1})-c_{t}\right) d t+\theta_{t}^{\prime} \sigma d W_{t}
$$

The pair $\left(\theta_{t}, c_{t}\right)$ is admissible for the initial wealth $x$ if the solution to (2), which is defined $P$-a.s., remains $P$-a.s. non-negative at all times. Let $\mathcal{A}^{P}(x)$ be the set of all admissible $(\theta, c)$ pairs for initial wealth $x$. In particular, the admissible set depends only on the equivalence class of $P$, namely we would get the same set under a $P^{*} \sim P$. Given a time horizon $T$, the agent is trying to choose $(\theta, c) \in \mathcal{A}^{P}(x)$, so as to maximize the expected utility from running consumption and terminal wealth:

$$
\sup _{(\theta, c) \in \mathcal{A}^{P}(x)} E\left[\int_{0}^{T} u\left(t, c_{t}\right) d t+u\left(T, X_{T}\right)\right] .
$$

This class of stochastic control problems is known under the name of Merton problem. It includes a number of specific cases, among which the infinite horizon planning. In fact, if $T=\infty, X_{\infty}:=\lim \sup _{t \rightarrow \infty} X_{t}$ and $u(\infty, \cdot)=0$ the above optimization problem becomes:

$$
\sup _{(\theta, c) \in \mathcal{A}^{P}(x)} E\left[\int_{0}^{\infty} u\left(t, c_{t}\right) d t\right] .
$$

The following sums up the requirements on $u$ needed in the present Section. 
Assumption 1 The utility function $u:(0, \infty) \times \mathbb{R}^{n} \rightarrow(-\infty, \infty)$ is jointly measurable. For fixed $t, u(t, x)$ is concave and increasing in $x$ and satisfies the Inada condition at $\infty$ :

$$
\lim _{x \rightarrow \infty} u^{\prime}(t, x)=0 .
$$

So far, the exposition is classical, and can be found in many textbooks. For an excellent didactic approach, the reader can consult the book [27, Chapter 1].

However, things change quite a bit if the agent is diffident about the (constant) estimates $\hat{\mu}$ and invertible matrix $\hat{\sigma}$, for the drift and volatility matrix of the risky assets, respectively.

\subsection{The robust framework}

Assume from now on that $\Omega$ is the $n$-dimensional Wiener space of continuous functions, with the natural filtration $\mathbf{F}=\left(\mathcal{F}_{t}\right)_{0 \leq t \leq T}$. Let $K$ be some fixed compact set of $n \times n$ symmetric and positive definite matrices, containing $\hat{\Sigma}$ and verifying a uniform ellipticity condition

$$
\Sigma \in K \Rightarrow \exists a>0 \text { s.t. } y^{\prime} \Sigma y \geq a^{2}\|y\|^{2} \forall y \in \mathbb{R}^{n} .
$$

The specification via $\Sigma$ for the ambiguity in the volatility is in line with empirical practice, as the (instantaneous) covariance matrix $\Sigma$ is the estimated object, and not the volatility $\sigma$. The Cholesky factorization offers a one-to-one correspondence between symmetric and positive definite matrices $\Sigma$ and lower triangular matrices $\sigma$ with positive diagonal elements, so that $\Sigma=\sigma \sigma^{\prime}$. Therefore, if $\left(\Sigma_{t}\right)_{t}=\left(\sigma_{t} \sigma_{t}^{\prime}\right)_{t}$, the plausible volatilities are:

$\mathcal{S}=\left\{\sigma\right.$ progr. meas. $\mid \sigma_{t}(\omega)$ is lower triangular, with positive diagonal and $\Sigma_{t}(\omega) \in K$ for all $\left.\omega, t\right\}$.

The uncertain drift is also assumed to be progressively measurable. For a given realization of the volatility $\sigma_{t}(\omega)$, or equivalently of the instantaneous covariance matrix $\Sigma_{t}(\omega)$, it is allowed to vary in

$$
U_{\epsilon}\left(\Sigma_{t}(\omega)\right)=\left\{u \in \mathbb{R}^{n} \mid(u-\hat{\mu})^{\prime} \Sigma_{t}^{-1}(\omega)(u-\hat{\mu}) \leq \epsilon^{2}\right\}
$$

that is, in an ellipsoid shaped by $\Sigma_{t}(\omega)$, centered at $\hat{\mu}$ and with constant radius $\epsilon \geq 0$. Let us denote the set of plausible processes by

$$
\Upsilon:=\left\{(\mu, \sigma) \text { progr. meas. } \mid \sigma \in \mathcal{S}, \mu_{t}(\omega) \in U_{\epsilon}\left(\Sigma_{t}(\omega)\right)\right\} .
$$

Let $S$ be the canonical process $(\Omega, \mathbf{F})$, namely $S_{t}(\omega)=\omega(t)$.

Definition 1 The plausible set $\mathcal{P}$ of probabilities is the set of $P$ s on $(\Omega, \mathbf{F})$ for which the process $S$ is the unique strong solution of the SDE

$$
d S_{t}=\operatorname{Diag}\left(S_{t}\right)\left(\mu_{t} d t+\sigma_{t} d W_{t}^{P}\right),
$$

in which $W^{P}$ denotes a $P$-Brownian motion, with $(\mu, \sigma) \in \Upsilon$.

Given the initial wealth $x>0$, the investment/consumption pair $(\theta, c)$ is called (robust) admissible if the measurability, integrability and nonnegativity assumptions made at the beginning of this section hold $P$-a.s. for all $P \in \mathcal{P}$. Namely,

$$
\mathcal{A}^{\mathrm{rob}}(x):=\cap_{P \in \mathcal{P}} \mathcal{A}^{P}(x) .
$$

So, the wealth $X$ has $P$-dynamics given by

$$
d X_{t}=\left(r X_{t}+\theta_{t}^{\prime}(\mu-r \mathbf{1})-c_{t}\right) d t+\theta_{t}^{\prime} \sigma_{t} d W_{t}^{P}
$$


Remark 1 The set $\mathcal{A}^{\mathrm{rob}}(x)$ is not empty. In fact, we now show it contains any strategy of the form:

$$
\theta_{t}=X_{t} \pi_{t}, c_{t}=\gamma_{t} X_{t}
$$

with $\gamma, \pi$ progr. measurable and uniformly bounded processes, respectively scalar and $\mathbb{R}^{n}$ valued. Under a fixed $P \in \mathcal{P}$, the wealth from the given strategy is

$$
d X_{t}=X_{t}\left[\left(r+\pi_{t}^{\prime}\left(\mu_{t}-r \mathbf{1}\right)-\gamma_{t}\right) d t+\pi_{t}^{\prime} \sigma_{t} d W_{t}^{P}\right]
$$

so that $X$ is the Doléans exponential

$$
x \mathcal{E}\left(\int_{0}^{\cdot}\left\{\left(r+\pi_{t}^{\prime}\left(\mu_{t}-r \mathbf{1}\right)-\gamma_{t}\right) d t+\pi_{t}^{\prime} \sigma_{t} d W_{t}^{P}\right\}\right) \geq 0 P-\text { a.s. }
$$

The ambiguity averse investor takes a prudential worst case approach, or alternatively she plays a game against the adverse market. The robust version of the Merton problem she faces is:

$$
V(0, x):=\sup _{(\theta, c) \in \mathcal{A}^{\mathrm{rob}}(x)} \inf _{P \in \mathcal{P}} E^{P}\left[\int_{0}^{T} u\left(t, c_{t}\right) d t+u\left(T, X_{T}\right)\right] .
$$

It is clear that more conservative portfolio choices are made when the uncertainty set $\Upsilon$ is larger, while an ambiguity-neutral investor sets $K=\{\hat{\Sigma}\}$ and $\epsilon$ equal to zero - thus facing a classical Merton problem under $\hat{P}$, as better detailed in the next Remark.

Remark 2 When $\sigma$ is non ambiguous, namely when $K=\{\hat{\Sigma}\}$, results on SDEs (see e.g. the book [26], Chap. 9, Sect. 2) imply that there exists a unique probability $\hat{P}$ on $(\Omega, \mathbf{F})$ such that the canonical process evolves as a diffusion with coefficients $(\hat{\mu}, \hat{\sigma})$. Then, the Girsanov Theorem ensures that all the probabilities in $\mathcal{P}$ are equivalent to $\hat{P}$ on $\mathcal{F}_{t}$ for all $t>0$. The unique probability $P \in \mathcal{P}$ corresponding to the coefficients $(\mu, \hat{\sigma})$ is obtained from a measure change wrt $\hat{P}$ given by the Doléans exponential $\mathcal{E}\left(\int_{0}^{\cdot} \varphi^{\mu} d W\right)$, where

$$
\varphi_{t}^{\mu}:=\hat{\sigma}_{t}^{-1}\left(\mu_{t}-\hat{\mu}\right) .
$$

Chen and Epstein [4] call the process $\varphi^{\mu}$ the market price of ambiguity. By the equivalence of the probabilities in $\mathcal{P}$, in this case

$$
\mathcal{A}^{\mathrm{rob}}(x)=\cap_{P \in \mathcal{P}} \mathcal{A}^{P}(x)=\mathcal{A}^{\hat{P}}(x) .
$$

Therefore, if additionally there is no ambiguity in the drift, the robust problem reduces to the classic Merton problem.

When ambiguity on the volatility is considered however, the situation abruptly changes. In fact, the quadratic variation process of the stochastic logarithm $L$ of $S$ under a fixed $P \in \mathcal{P}$ is:

$$
\langle L\rangle_{t}=\int_{0}^{t} \Sigma_{s} d s
$$

and is unique $P$-almost surely. So, if under two probabilities $P_{1}, P_{2} \in \mathcal{P}$ the process $L$ has different quadratic variation, in the sense that the progressively measurable set

$$
A=\left\{(\omega, t) \mid \Sigma_{t}^{1}(\omega) \neq \Sigma_{t}^{2}(\omega)\right\}
$$

has positive measure wrt $d P_{1} d t$ and $d P_{2} d t$, then neither $P_{1} \ll P_{2}$ nor $P_{2} \ll P_{1}$. In the extreme case in which the set $A$ coincides with $\Omega \times[0, T]$, then $P_{1}$ and $P_{2}$ are mutually singular. 
Lemma 1 The value function $V(0, \cdot)$ of the general robust problem (6) is monotone increasing and concave on $\mathbb{R}_{+}$.

Proof - Monotonicity of the utility $u$ and the implication $0<x^{1} \leq x^{2} \Rightarrow \mathcal{A}^{\mathrm{rob}}\left(x^{1}\right) \subseteq$ $\mathcal{A}^{\mathrm{rob}}\left(x^{2}\right)$ give the monotonicity of the value function.

In fact, suppose $x^{1} \leq x^{2}$ and fix an arbitrary $\left(\theta^{1}, c^{1}\right) \in \mathcal{A}^{\mathrm{rob}}\left(x^{1}\right)$. If $X^{1}$ denotes the wealth from this plan, $X_{t}^{2}=\left(x^{2}-x^{1}\right) e^{r t}+X^{1}(t)$ is the wealth obtained by putting the extra initial endowment in the safe asset. The wealth process $X^{2}$ verifies (5) under any $P$ with the plan $\left(\theta^{1}, c^{1}\right)$, is clearly nonnegative as $X^{2} \geq X^{1}$, so that $\left(\theta^{1}, c^{1}\right)$ belongs to $\mathcal{A}^{\text {rob }}\left(x^{2}\right)$. The conclusion follows from:

$$
\begin{aligned}
V(0, x) & \leq \sup _{\left(\theta^{1}, c^{1}\right) \in \mathcal{A}^{\mathrm{rob}}\left(x^{1}\right)} \inf _{P \in \mathcal{P}} E^{P}\left[\int_{0}^{T} u\left(t, c_{t}^{1}\right) d t+u\left(T, X_{T}^{2}\right)\right] \\
& \leq \sup _{(\theta, c) \in \mathcal{A}^{\mathrm{rob}}\left(x^{2}\right)} \inf _{P \in \mathcal{P}} E^{P}\left[\int_{0}^{T} u\left(t, c_{t}\right) d t+u\left(T, X_{T}\right)\right]
\end{aligned}
$$

in which the first inequality holds by monotonicity of $u(T, \cdot)$.

- Let $x=\alpha x_{1}+(1-\alpha) x_{2}$, with $\alpha \in[0,1]$, and positive $x^{1}, x^{2}$. By linearity of the wealth equation, $\mathcal{A}^{\mathrm{rob}}(x) \supseteq \alpha \mathcal{A}^{\mathrm{rob}}\left(x^{1}\right)+(1-\alpha) \mathcal{A}^{\mathrm{rob}}\left(x^{2}\right)$. If $X^{i}$ is an admissible wealth in $\mathcal{A}^{\mathrm{rob}}\left(x^{i}\right)$, from the investment-consumption plan $\left(\theta^{i}, c^{i}\right), i=1,2$, then

$$
\begin{aligned}
V(0, x) \geq & \sup _{\left(\theta^{1}, c^{1}\right) \in \mathcal{A}^{\mathrm{rob}}\left(x^{1}\right),\left(\theta^{2}, c^{2}\right) \in \mathcal{A}^{\mathrm{rob}}\left(x_{2}\right)} \inf _{P \in \mathcal{P}} E^{P} \\
& E^{P}\left[\int_{0}^{T} u\left(s, \alpha c_{s}^{1}+(1-\alpha) c_{s}^{2}\right) d s+u\left(T, \alpha X^{1}(T)+(1-\alpha) X^{2}(T)\right)\right] .
\end{aligned}
$$

By concavity of $u$ and the fact that the infimum of a sum is greater or equal to the sum of the infima, we get that the expression above is minorized by

$$
\begin{aligned}
& \sup _{\left(\theta^{1}, c^{1}\right) \in \mathcal{A}^{\mathrm{rob}}\left(x^{1}\right),\left(\theta^{2}, c^{2}\right) \in \mathcal{A}^{\mathrm{rob}}\left(x_{2}\right)}\left\{\alpha \inf _{P \in \mathcal{P}} E^{P}\left[\int_{0}^{T} u\left(s, c_{s}^{1}\right) d s+u\left(T, X^{1}(t)\right)\right]\right. \\
& \left.+(1-\alpha) \inf _{P \in \mathcal{P}} E^{P}\left[\int_{0}^{T} u\left(s, c_{s}^{2}\right) d s+u\left(T, X^{2}(t)\right)\right]\right\} .
\end{aligned}
$$

This supremum is in turn equal to the sum of the suprema, since the variables separate. So,

$$
V\left(0, \alpha x^{1}+(1-\alpha) x^{2}\right) \geq \alpha V\left(0, x^{1}\right)+(1-\alpha) V\left(0, x^{2}\right) .
$$

The solution of the robust problem (6) is based on the next version of the verification theorem.

\section{Theorem 1 Suppose that:}

1. there exists a function $v:[0, T] \times \mathbb{R}^{+} \rightarrow \mathbb{R}$, which is continuous on $[0, T] \times \mathbb{R}^{+}$and $C^{1,2}$ on $[0, T) \times \mathbb{R}^{+}$, verifying $v(T, \cdot)=u(T, \cdot)$;

2. for any $(\theta, c) \in \mathcal{A}^{r o b}(x)$ there exists an optimal solution $P^{(\theta, c)}$ of the inner minimization in (6), such that

$$
Y_{t}=Y_{t}^{(\theta, c)}=v\left(t, X_{t}\right)+\int_{0}^{t} u\left(s, c_{s}\right) d s
$$

is a $P^{(\theta, c)}$-supermartingale; 
3. there exist some $(\bar{\theta}, \bar{c}) \in \mathcal{A}^{\mathrm{rob}}(x)$ such that the corresponding $\bar{Y}$ is a $P^{(\bar{\theta}, \bar{c})}$-martingale.

Then $(\bar{\theta}, \bar{c})$ is optimal for the problem $(6)$ and $v(0, x)$ coincides with $V(0, x)$.

Proof The proof is a simple modification of the Davis-Varaiya Martingale Principle of Optimal Control [27, Theorem1.1]. In fact, by the supermartingale property of $Y$ under $P^{(\theta, c)}$, and by $V(T, \cdot)=u(T, \cdot)$, we have:

$$
E^{P^{(\theta, c)}}\left[Y_{T}\right]=E^{P^{(\theta, c)}}\left[\int_{0}^{T} u\left(s, c_{s}\right) d s+u\left(T, X_{T}\right)\right] \leq Y_{0}=v(0, x) .
$$

Taking the supremum over $\mathcal{A}^{\mathrm{rob}}(x)$ gives $V(0, x)=\sup _{\mathcal{A}^{\mathrm{rob}}(x)} E^{P^{(\theta, c)}}\left[\int_{0}^{T} u\left(s, c_{s}\right) d s+\right.$ $\left.u\left(T, X_{T}\right)\right] \leq v(0, x)$. Since by assumption for some $(\bar{\theta}, \bar{c})$ the process $\bar{Y}$ is a martingale under $P^{(\bar{\theta}, \bar{c})}$, then $E^{P^{(\bar{\theta}, \bar{c})}}\left[\bar{Y}_{T}\right]=Y_{0}=v(0, x)$ and the conclusions immediately follow.

Now, the usage of the verification theorem to solve the ambiguity-averse investor's problem is quite intuitive. Given a specific utility function, one looks for a function $v$ which satisfies the premises of the theorem. Using the Itō's formula under $P^{(\theta, c)}$ then, any process $Y$ as in (7) verifies the SDE:

$$
d Y_{t}=\left\{u\left(t, c_{t}\right)+v_{t}+v_{x}\left(r X_{t}+\theta_{t}^{\prime}\left(\mu_{t}-r \mathbf{1}\right)-c_{t}\right)+\frac{1}{2} \theta_{t}^{\prime} \Sigma_{t} \theta_{t} v_{x x}\right\} d t+v_{x} \theta_{t}^{\prime} \sigma_{t} d W_{t}^{P^{(\theta, c)}}
$$

If $Y$ has to be a supermartingale under every $P^{(\theta, c)}$, and a martingale for some $P^{(\bar{\theta}, \bar{c})}$, the supremum over $(\theta, c) \in \mathbb{R}^{n} \times \mathbb{R}^{+}$of the infimum over $\Sigma \in K, \mu \in U_{\epsilon}(\Sigma)$ of the drift of $Y$ must be equated to zero. Thus, a sup-inf nonlinear PDE arises:

$$
\sup _{(\theta, c) \in \mathbb{R}^{n} \times \mathbb{R}^{+}} \inf _{\Sigma \in K, \mu \in U_{\epsilon}(\Sigma)}\left[u(t, c)+v_{t}+v_{x}\left(r x+\theta^{\prime}(\mu-r \mathbf{1})-c\right)+\frac{1}{2} \theta^{\prime} \Sigma \theta v_{x x}\right]=0,
$$

which is of the Hamilton-Jacobi-Bellman-Isaacs type (HJBI for short, see [7] for more information).

Lemma 2 The map $\theta \mapsto M(\theta):=\max \left\{\theta^{\prime} \Sigma \theta \mid \Sigma \in K\right\}$ is continuous.

Proof The constant correspondence

$$
\varphi: \mathbb{R}^{n} \rightarrow \mathcal{M}(n \times n), \quad \varphi(\theta):=K
$$

is clearly compact valued and continuous. Then, the continuous function

$$
f: \operatorname{Graph}(\varphi) \rightarrow \mathbb{R}, \quad f(\theta, \Sigma):=\theta^{\prime} \Sigma \theta
$$

has a continuous max function $M(\theta)=\max _{K} f(\theta, \Sigma)$ (see e.g. the book [2, Lemmata 17.29 and 17.30]).

By Lemma 1, the value function $V$ is increasing and concave in the wealth $x$. Thus we naturally restrict to candidates $v$ with the same properties, for which the following result holds.

Proposition 1 Under Assumption 1, the supremum and the infimum in the HJBI equation (9) are attained for any $v \in C^{1,2}$ on $(0, T) \times \mathbb{R}^{+}$verifying $v_{x}>0, v_{x x}<0$. 
Proof We first minimize the drifts for $\Sigma$ fixed:

$$
\min _{\mu \in \mathbb{R}^{n}}\left\{\theta^{\prime} \mu:(\mu-\hat{\mu})^{\prime} \Sigma^{-1}(\mu-\hat{\mu}) \leq \epsilon^{2}\right\},
$$

which is a simple exercise in Karush-Kuhn-Tucker necessary and sufficient conditions. When $\theta \neq 0$, the optimal solution is

$$
\mu(\theta)=\hat{\mu}-\epsilon \frac{\Sigma \theta}{\sqrt{\theta^{\prime} \Sigma \theta}} .
$$

Substituting it back in the Hamiltonian of the HJBI equation, we get

$$
\sup _{(\theta, c) \in \mathbb{R}^{n} \times \mathbb{R}^{+} \Sigma \in K} \inf _{\Sigma \in}\left[u(t, c)+v_{t}+v_{x}\left(r x+\theta^{\prime}(\hat{\mu}-r \mathbf{1})-\epsilon \sqrt{\theta^{\prime} \Sigma \theta}-c\right)+\frac{1}{2} \theta^{\prime} \Sigma \theta v_{x x}\right],
$$

which covers also the case $\theta=0$. The minimization

$$
\inf _{\Sigma \in K}\left(-v_{x} \epsilon \sqrt{\theta^{\prime} \Sigma \theta}+\frac{v_{x x}}{2} \theta^{\prime} \Sigma \theta\right)
$$

is then immediate. In fact, set $s=\sqrt{\theta^{\prime} \Sigma \theta}$. Then, the above is the restriction of the concave parabola

$$
y(s)=-\epsilon v_{x} s+\frac{1}{2} v_{x x} s^{2}
$$

to a compact subset of the positive axis. By the signs considerations on the derivatives of $v$, the vertex has negative abscissa, and the minimum is reached for the maximum $s$. Therefore,

$$
\operatorname{argmax}_{K} \theta^{\prime} \Sigma \theta \quad \text { is the set of optimal } \Sigma s .
$$

Keeping the notation of the previous Lemma, we get

$$
\sup _{(\theta, c) \in \mathbb{R}^{n} \times \mathbb{R}^{+}}\left[u(t, c)+v_{t}+v_{x}\left(r x+\theta^{\prime}(\hat{\mu}-r \mathbf{1})-\epsilon \sqrt{M(\theta)}-c\right)+\frac{1}{2} M(\theta) v_{x x}\right]=0 .
$$

The maximization can be split into the sum of:

1. $\sup _{c \in \mathbb{R}^{+}}\left(u(t, c)+v_{t}-c v_{x}\right)$. This is attained by some $\bar{c}$ thanks to concavity and regularity of $u$, to $v_{x}>0$ and to the Inada condition at $+\infty$ on $u$, which implies $\lim _{c \rightarrow+\infty}[u(t, c)+$ $\left.v_{t}-c v_{x}\right]=-\infty$.

2. $\sup _{\theta \in \mathbb{R}^{n}}\left[v_{x}\left(r x+\theta^{\prime}(\hat{\mu}-r \mathbf{1})-\epsilon \sqrt{M(\theta)}\right)+\frac{1}{2} M(\theta) v_{x x}\right]$. The signs of $v_{x x}$ and $v_{x}$ and the uniform ellipticity assumption (3) ensures coercivity of this objective function when $\|\theta\| \rightarrow+\infty$ :

$$
\begin{gathered}
v_{x}\left(r x+\theta^{\prime}(\hat{\mu}-r \mathbf{1})-\epsilon \sqrt{M(\theta)}\right)+\frac{1}{2} M(\theta) v_{x x} \leq \\
v_{x}\left(r x+\theta^{\prime}(\hat{\mu}-r \mathbf{1})-\epsilon a\|\theta\|\right) \\
+\frac{1}{2} a^{2} v_{x x}\|\theta\|^{2}
\end{gathered}
$$

The sup is then attained by some $\bar{\theta}$, since the objective function is continuous.

The techniques employed to solve (12) for a specific utility rely, as usual, on educated guesses at the form of the solution. We conclude this Section with a remark on the validity of the above results for more general uncertainty sets. 
Remark 3 The Hamiltonian in the robust PDE, thought as a function of the agent/market controls, is a continuous function $G((\theta, c),(\mu, \Sigma))$. As long as the couple $(\mu, \Sigma)$ varies on a compact $\mathcal{K}$, with uniformly elliptic projection on the $\Sigma$ variable, mutatis mutandis the arguments in the above Lemma and Proposition still apply. In particular,

$$
\min _{(\mu, \Sigma) \in \mathcal{K}} G((\theta, c),(\mu, \Sigma))
$$

is a continuous function of $(\theta, c)$. The max over the strategies is then attained thanks to the Inada condition on $u$ and to uniform ellipticity of the matrices $\Sigma$.

\section{The robust CRRA utility problem with constant, non ambiguous $\Sigma$}

In this Section there is no uncertainty on the (constant) square volatility matrix, namely $K=\{\hat{\Sigma}\}$. Lack of uncertainty in the volatility is interesting per se since mean returns are subject to imprecision to a much higher extent than volatilities. So, this case may be seen as a first approximation to the general problem.

To avoid notation overload, and for the next usage in Sect. 4, we drop the hat over $\hat{\Sigma}$. The set $\Upsilon$ in (4) here is just

$$
\left\{(\mu, \sigma) \mid \mu \text { progr. meas. and } \mu_{t}(\omega) \in U_{\epsilon}(\Sigma) \text { for all } \omega\right\} .
$$

We call $\mathcal{P}_{\Sigma}$ the set of plausible probabilities (Definition 1), to stress that $\Sigma$ is fixed.

\subsection{The infinite horizon planning}

\subsubsection{Resolution of the HJBI equation}

Let us assume the investor has CRRA utility from intertemporal consumption. Fix the positive constants $\rho$ and $R$, modeling the time impatience rate and relative risk aversion respectively. Then, the utility is

1. $u(t, x)=e^{-\rho t} \frac{x^{1-R}}{1-R}$, with $R \neq 1$, or

2. $u(t, x)=e^{-\rho t} \ln x$ if $R=1$.

In the infinite horizon case, we wish to find the solution of:

$$
V_{\Sigma}(0, x)=\sup _{(\theta, c) \in \mathcal{A}^{\mathrm{rob}}(x)} \inf _{P \in \mathcal{P}_{\Sigma}} E^{P}\left[\int_{0}^{\infty} u\left(s, c_{s}\right) d s\right],
$$

Assume for the moment that this problem is finite valued, and that both the inner infimum (for a fixed $\left.(\theta, c) \in \mathcal{A}^{\mathrm{rob}}(x)\right)$ and the outer supremum are attained. Structural properties imply, exactly as in the classic cases, that a guess at the value function takes the form

1. $v(t, x)=\left(\gamma_{\epsilon}\right)^{-R} e^{-\rho t} \frac{x^{1-R}}{1-R}, R \neq 1$ and

2. $v(t, x)=e^{-\rho t}\left(\frac{\ln x}{\rho}+k_{\epsilon}\right), R=1$,

where $\gamma_{\epsilon}$ and $k_{\epsilon}$ are positive constants to be determined. We use $\epsilon$ as subscript to highlight the dependence on the radius of drift ambiguity $\epsilon$. The candidate $v$ verifies the conditions in Proposition 1, so that the optima are attained in the HJBI equation (9). Substituing the optimal $\mu(\theta)$ as in (10), the residual optimization is given by (12). Maximizing over $c$ trivially results in 
1. $\bar{c}=\gamma_{\epsilon} x$ in the power case

2. $\bar{c}=\rho x$ in the logarithmic case,

with optimal value

$$
\max _{c}\left\{u(t, c)-c v_{x}\right\}=e^{-\rho t} \psi_{\epsilon}(x, R),
$$

in which we set

1. $\psi_{\epsilon}(x, R)=\frac{R}{1-R}\left(\gamma_{\epsilon} x\right)^{1-R}, R \neq 1$

2. $\psi_{\epsilon}(x, 1)=\ln \rho x-1$ for $R=1$.

The function to be maximized becomes

$$
\max _{\theta}\left[e^{-\rho t} \psi_{\epsilon}(x, R)+v_{t}+v_{x}\left(r x+\theta^{\prime}(\hat{\mu}-r \mathbf{1})-\epsilon \sqrt{\theta^{\prime} \Sigma \theta}\right)+\frac{1}{2} \theta^{\prime} \Sigma \theta v_{x x}\right] .
$$

which is concave in $\theta$, and smooth in $\mathbb{R}^{n} \backslash\{0\}$. The first order conditions are thus necessary and sufficient for optimality in $\theta \neq 0$. So, by equating the gradient to zero we obtain:

$$
\theta(s)=\frac{-s v_{x}}{s v_{x x}-v_{x} \epsilon} \Sigma^{-1}(\hat{\mu}-r \mathbf{1}),
$$

where $s:=\sqrt{\theta^{\prime} \Sigma \theta}$. We are left with

$$
s^{2}=\theta(s)^{\prime} \Sigma \theta(s)
$$

Set

$$
H:=\sqrt{(\hat{\mu}-r \mathbf{1})^{\prime} \Sigma^{-1}(\hat{\mu}-r \mathbf{1})}, \quad H_{\epsilon}:=H-\epsilon
$$

The above equation has a positive root, given by:

$$
\bar{s}=-\frac{v_{x} H_{\epsilon}}{v_{x x}}
$$

if and only if $H_{\epsilon}>0$. If $H_{\epsilon} \leq 0$, the optimal solution (which exists by Proposition 1) is necessarily $\bar{\theta}=0$. Finally, if $H_{\epsilon}^{+}$denotes the positive part of $H_{\epsilon}$, the following is a compact way of writing the optimal solution in both the power and logarithmic case:

$$
\bar{\theta}=x \frac{H_{\epsilon}^{+}}{R H} \Sigma^{-1}(\hat{\mu}-r \mathbf{1})
$$

The value of the constants $\gamma_{\epsilon}, k_{\epsilon}$ is found by substituting these $\bar{c}$ and $\bar{\theta}$ back into (12) and solving the equation. Straightforward calculations result in:

1. $\gamma_{\epsilon}=\frac{\rho+(R-1)\left(r+\frac{1}{2} \frac{\left(H_{\epsilon}^{+}\right)^{2}}{R}\right)}{R}$ and

2. $k_{\epsilon}=\frac{1}{\rho^{2}}\left[\rho \ln \rho+r-\rho+\frac{\left(H_{\epsilon}^{+}\right)^{2}}{2}\right]$

which for $\epsilon=0$ fall back to the constants $\gamma_{0}=\frac{\rho+(R-1)\left[r+\frac{1}{2} \frac{H^{2}}{R}\right]}{R}, k_{0}=\frac{1}{\rho^{2}}\left[\rho \ln \rho+r-\rho+\frac{H^{2}}{2}\right]$ of the classic cases.

The well-posedness conditions and the verification that $V_{\Sigma}(0, x)=v(0, x)$ are the objects of the next subsection. 


\subsubsection{The verification and comparison with the classic Merton problem}

If the drift process $\mu$ is deterministic and bounded, there exists only one corresponding measure $P^{\mu}=P^{\mu, \Sigma}$ on the canonical space. For the class of bounded (wealth) proportion investment-consumption plan, the minimal drift $\mu(\theta)$ in (10) is deterministic. The corresponding probability $P^{\mu(\theta)}$ belongs to $\mathcal{P}_{\Sigma}$ and is indeed an optimal control for the adverse market.

Lemma 3 Let $(\theta, c)$ be a plan of the form

$$
\theta_{t}=X_{t} \pi_{t}, \quad c_{t}=X_{t} \lambda_{t}
$$

in which $\pi$ and $\lambda \geq 0$ are deterministic and bounded. Then, $(\theta, c) \in \mathcal{A}^{\mathrm{rob}}(x)$ and the probability $P^{\mu(\theta)}$ corresponding to the drift

$$
\mu_{t}(\theta)=\mu_{t}(\pi)=\hat{\mu}-\epsilon \frac{\Sigma \pi_{t}}{\sqrt{\pi_{t}^{\prime} \Sigma \pi_{t}}} .
$$

(where we adopt the convention $\frac{0}{0}=0$ ) verifies

$$
\min _{P \in \mathcal{P}_{\Sigma}} E^{P}\left[\int_{0}^{\infty} e^{-\rho t} u\left(s, X_{s} \lambda_{s}\right) d s\right]=E^{P^{\mu(\theta)}}\left[\int_{0}^{\infty} e^{-\rho t} u\left(s, X_{s} \lambda_{s}\right) d s\right]:=m(\theta, c)
$$

The minimal expectation $m(\theta, c)$ is given by

1. $R \neq 1$ :

$$
\begin{aligned}
m(\theta, c)= & \frac{x^{1-R}}{1-R} \int_{0}^{\infty} e^{-\rho t} \lambda_{t}^{1-R} \\
& \quad \times \exp \left(\int_{0}^{t}(1-R)\left(r-\lambda_{s}+\pi_{s}^{\prime}(\hat{\mu}-r \mathbf{1})-\epsilon \sqrt{\pi_{s}^{\prime} \Sigma \pi_{s}}-\frac{R}{2} \pi_{s}^{\prime} \Sigma \pi_{s}\right) d s\right) d t
\end{aligned}
$$

2. $R=1$ :

$$
\begin{aligned}
m(\theta, c)= & \frac{\ln x}{\rho}+\int_{0}^{\infty} d t e^{-\rho t} \\
& \times\left(\ln \lambda_{t}+\int_{0}^{t}\left(r-\lambda_{s}+\pi_{s}^{\prime}(\hat{\mu}-r \mathbf{1})-\epsilon \sqrt{\pi_{s}^{\prime} \Sigma \pi_{s}}-\frac{1}{2} \pi_{s}^{\prime} \Sigma \pi_{s}\right) d s\right) .
\end{aligned}
$$

As a consequence,

$$
V_{\Sigma}(0, x) \geq m(\theta, c),
$$

and the dynamics of the wealth generated by $(\theta, c)$ under $P^{\mu(\theta)}$ are

$$
d X_{t}=X_{t}\left(r+\pi_{t}^{\prime}\left(\hat{\mu}-\epsilon \frac{\Sigma \pi_{t}}{\sqrt{\pi_{t}^{\prime} \Sigma \pi_{t}}}-r \mathbf{1}\right)-\lambda_{t}\right) d t+X_{t} \pi_{t}^{\prime} \sigma d W_{t}^{P^{\mu(\theta)}} .
$$

Proof By Remark 1, the given plan is admissible and the solution to the wealth equation under a $P \in \mathcal{P}_{\Sigma}$ is

$$
X_{t}=x \exp \left(\int_{0}^{t}\left(r-\lambda_{s}+\pi_{s}^{\prime}\left(\mu_{s}-r \mathbf{1}\right)\right) d s \exp \left(\int_{0}^{t} \pi_{s}^{\prime} \sigma d W_{s}^{P}-\frac{1}{2} \int_{0}^{t} \pi_{s}^{\prime} \Sigma \pi_{s} d s\right)>0\right.
$$


Consider then the power and logarithmic cases separately:

- Power case. Applying the stochastic Fubini-Tonelli theorem,

$$
E^{P}\left[\int_{0}^{\infty} e^{-\rho t} \frac{\left(X_{t} \lambda_{t}\right)^{1-R}}{1-R} d t\right]=\int_{0}^{\infty} e^{-\rho t} \lambda_{t}^{1-R} \frac{E^{P}\left[X_{t}^{1-R}\right]}{1-R} d t .
$$

The last term is indeed minimized by the constant drift $\mu(\theta)$. In fact, $\frac{E^{P}\left[X_{t}^{1-R}\right]}{1-R}$ equals

$$
\begin{aligned}
& x^{1-R} \exp \left((1-R)\left(\int_{0}^{t}\left\{\left(r\left(1-\pi_{s}^{\prime} \mathbf{1}\right)-\lambda_{s}-\frac{R}{2} \pi_{s}^{\prime} \Sigma \pi_{s}\right\} d s\right)\right)\right. \\
& E^{P}\left[\frac{1}{1-R} \exp \left((1-R) \int_{0}^{t} \pi_{s}^{\prime} \mu_{s} d s\right)\right] .
\end{aligned}
$$

By (the proof of) Proposition 1, the random variable inside the expectation is pointwisely minimized by the deterministic $\mu(\theta)$ given by $\mu_{t}(\theta)=\mu_{t}(\pi)=\hat{\mu}-\epsilon \frac{\Sigma \pi_{t}}{\sqrt{\pi_{t}^{\prime} \Sigma \pi_{t}}}$. Therefore,

$$
\begin{aligned}
\frac{E^{P}\left[\exp \left((1-R) \int_{0}^{t} \pi_{s}^{\prime} \mu_{s} d s\right)\right]}{1-R} & \geq \frac{1}{1-R} \exp \left((1-R) \int_{0}^{t} \pi_{s}^{\prime} \mu_{s}(\theta) d s\right) \\
& =\frac{E^{P^{\mu(\theta)}}\left[\exp \left((1-R) \int_{0}^{t} \pi_{s}^{\prime} \mu_{s}(\theta) d s\right)\right]}{1-R}
\end{aligned}
$$

which gives minimality of $P^{\mu(\theta)}$. Substituting the values, we get the expression in the first item of the statement.

- Log case. Proceeding as above,

$$
E^{P}\left[\int_{0}^{\infty} e^{-\rho t} \ln \left(X_{t} \lambda_{t}\right) d t\right]=\int_{0}^{\infty} d t e^{-\rho t}\left(\ln \lambda_{t}+E^{P}\left[\ln X_{t}\right]\right) .
$$

The expectation on the right is $E^{P}\left[\ln x+\int_{0}^{t}\left(r-\lambda_{s}+\pi_{s}^{\prime}\left(\mu_{s}-r \mathbf{1}\right)-\frac{1}{2} \pi_{s}^{\prime} \Sigma \pi_{s}\right) d s\right]$, which once again is minimized pointwisely by the deterministic $\mu(\theta)$, so the conclusion follows.

Corollary 1 The candidate optimal wealth $\bar{X}$ has $\bar{P}:=P^{\mu(\bar{\theta})}$ dynamics given by:

$$
d \bar{X}_{t}=\bar{X}_{t}\left(r+\pi_{\epsilon}^{\prime}(\hat{\mu}-r \mathbf{1})-\epsilon \sqrt{\pi_{\epsilon}^{\prime} \Sigma \pi_{\epsilon}}-\bar{\lambda}_{R}\right) d t+\bar{X}_{t} \pi_{\epsilon}^{\prime} \sigma d W_{t}^{\bar{P}}
$$

where the consumption rate $\bar{\lambda}_{R}$ equals $\gamma_{\epsilon}$ if $R \neq 1$, and $\rho$ if $R=1$.

Proof Just substitute the expression of the candidate optimal controls in the wealth equation (17).

Theorem 2 (Power case) The infinite-horizon robust Merton problem, with power utility and initial endowment $x>0$, under ellipsoidal ambiguity of mean returns:

$$
V_{\Sigma}(0, x)=\sup _{(\theta, c) \in \mathcal{A}^{\mathrm{rob}}(x)} \inf _{P \in \mathcal{P}_{\Sigma}} E^{P}\left[\int_{0}^{\infty} e^{-\rho t} \frac{c_{t}^{1-R}}{1-R} d t\right],
$$

is finite valued if and only if

$$
\gamma_{\epsilon}=\frac{\rho+(R-1)\left(r+\frac{1}{2} \frac{\left(H_{\epsilon}^{+}\right)^{2}}{R}\right)}{R}>0
$$

with $H_{\epsilon}=\sqrt{(\hat{\mu}-r \mathbf{1})^{\prime} \Sigma^{-1}(\hat{\mu}-r \mathbf{1})}-\epsilon$ as in (14). In case $\gamma_{\epsilon}>0$ : 
- The optimal value is

$$
V_{\Sigma}(0, x)=v(0, x)=\gamma_{\epsilon}^{-R} \frac{x^{1-R}}{1-R}
$$

- The optimal agent controls are:

$$
\bar{\theta}_{t}=\pi_{\epsilon} \bar{X}_{t}, \quad \bar{c}_{t}=\gamma_{\epsilon} \bar{X}_{t},
$$

with optimal portfolio proportions vector given by

$$
\pi_{\epsilon}:=\frac{H_{\epsilon}^{+}}{R H} \Sigma^{-1}(\hat{\mu}-r \mathbf{1})
$$

The optimal portfolio $\pi_{\epsilon}$ is therefore null if and only if $H_{\epsilon} \leq 0$ or, equivalently, $r \mathbf{1}$ is a plausible drift.

- The optimal adverse control is the probability $\bar{P}$ under which $S$ evolves with constant instantaneous covariance $\Sigma$ and drift

$$
\bar{\mu}:= \begin{cases}\hat{\mu}-\epsilon \frac{\Sigma}{\sqrt{\pi_{\epsilon}^{\prime} \Sigma \pi_{\epsilon}}} \pi_{\epsilon} & \text { if } r \mathbf{1} \notin U_{\epsilon}(\Sigma) \\ r \mathbf{1} & \text { otherwise }\end{cases}
$$

- The optimal drift $\bar{\mu}$ pointwisely realizes the worst market Sharpe Ratio among the plausible models $\mathcal{P}_{\Sigma}$ :

$$
\min _{P \in \mathcal{P}_{\Sigma}} \sqrt{\left(\mu_{t}-r \mathbf{1}\right)^{\prime} \Sigma^{-1}\left(\mu_{t}-r \mathbf{1}\right)}=\text { const }=H_{\epsilon}^{+}
$$

- The optimal wealth process has $\bar{P}$ dynamics given by

$$
\bar{X}_{t}=x \exp \left(\pi_{\epsilon} \sigma W_{t}^{\bar{P}}+\left(r+\frac{\left(H_{\epsilon}^{+}\right)^{2}(2 R-1)}{2 R^{2}}-\gamma_{\epsilon}\right) t\right)
$$

when $\pi_{\epsilon} \neq 0$, and is the deterministic $\bar{X}_{t}=x \exp \left(\left(r-\gamma_{\epsilon}\right) t\right)$ otherwise.

- The minimax equality holds:

$$
V_{\Sigma}(0, x)=\inf _{P \in \mathcal{P}_{\Sigma}} \sup _{(\theta, c) \in \mathcal{A}^{\mathrm{rob}}(x)} E^{P}\left[\int_{0}^{\infty} e^{-\rho t} \frac{c_{t}^{1-R}}{1-R} d t\right]
$$

and $((\bar{\theta}, \bar{c}), \bar{P})$ is a saddle point for the agent-market game.

Proof The proof is split into several steps.

- If $\gamma_{\epsilon} \leq 0$ then $V_{\Sigma}(0, x)=\infty$. Note first that this case can only happen when $0<R<1$. The proof here closely follows the lines of [27, Section1.6].

(a) Assume $\gamma_{\epsilon}<0$. Then, consider a constant proportion plan:

$$
\theta_{t}=\pi X_{t}, \quad c_{t}=\lambda X_{t} \quad \text { with } \quad \lambda>0
$$

By Lemma 3, which holds also when the candidate from (15) is $\pi_{\epsilon}=0$,

$$
\begin{aligned}
& V_{\Sigma}(0, x) \geq E^{P^{\mu(\theta)}}\left[\int_{0}^{\infty} e^{-\rho t} \frac{\lambda^{1-R}}{1-R}\left(X_{t}\right)^{1-R} d t\right]=\frac{x^{1-R}}{1-R} \lambda^{1-R} \\
& \quad \times \int_{0}^{\infty} \exp \left[t\left(-\rho+(1-R)\left(r+\pi^{\prime}(\hat{\mu}-r \mathbf{1})-\epsilon \sqrt{\pi^{\prime} \Sigma \pi}-\lambda-\frac{R}{2} \pi^{\prime} \Sigma \pi\right)\right)\right] d t
\end{aligned}
$$


If there exists some $(\pi, \lambda)$ for which the exponent is positive, then the integral diverges and the value function is infinite. For fixed $\lambda$, the maximum over $\pi$ in the exponent is attained for $\bar{\pi}=\pi_{\epsilon}$, and the value is

$$
-\rho+(1-R)\left(r+\frac{\left(H_{\epsilon}^{+}\right)^{2}}{2 R}-\lambda\right)=-R \gamma_{\epsilon}-\lambda(1-R),
$$

which is positive for $\lambda$ small enough.

(b) If $\gamma_{\epsilon}=0$, take $\theta_{t}=X_{t} \pi_{\epsilon}$, and $c_{t}=\frac{k}{1+t} X_{t}$ for some constant $k>0$. Using again Lemma 3, and collecting terms,

$$
\begin{aligned}
V_{\Sigma}(0, x) & \geq \frac{x^{1-R}}{1-R} \int_{0}^{\infty} e^{-\gamma_{\epsilon} R t} \frac{k^{1-R}}{(1+t)^{1-R}} e^{-(1-R) \int_{0}^{t} \frac{k}{1+s} d s} d t \\
& =\frac{x^{1-R}}{1-R} \int_{0}^{\infty} \frac{k^{1-R}}{(1+t)^{1-R}} e^{-(1-R) \int_{0}^{t} \frac{k}{1+s} d s} d t
\end{aligned}
$$

Now, $e^{-(1-R) \int_{0}^{t} \frac{k}{1+s} d s}=e^{-(1-R) k \ln (1+t)}=\frac{1}{(1+t)^{k(1-R)}}$ when $t \rightarrow \infty$. Therefore, the integrand is asymptotic to $(1+t)^{-(k+1)(1-R)}$ and hence the integral diverges if e.g., $k=\frac{R}{1-R}$.

- If $\gamma_{\epsilon}>0$, then the optimal value/controls/wealth are as given in the statement of this proposition.

1. Low ambiguity case $H_{\epsilon}>0$, or equivalently $r \mathbf{1} \notin U_{\epsilon}(\Sigma)$.

- Martingale property of the candidate optimal solution. Solving (18) for the candidate optimal wealth gives the process $\bar{X}$ in (19). The diffusion $\bar{Y}$

$$
\bar{Y}_{t}:=v\left(t, \bar{X}_{t}\right)+\int_{0}^{t} u\left(s, \bar{c}_{s}\right) d s=\gamma_{\epsilon}^{-R} e^{-\rho t} \frac{\bar{X}_{t}^{1-R}}{1-R}+\int_{0}^{t} e^{-\rho s}\left(\gamma_{\epsilon} \bar{X}_{s}\right)^{1-R} d s
$$

has zero drift term by construction, and therefore it is a uniformly integrable martingale as soon as appropriate integrability conditions hold. In our case, this is immediate as $\bar{X}_{t}^{1-R}$ is a deterministic scaling of a $\bar{P}$-Geometric Brownian motion.

- Optimality of $((\bar{\theta}, \bar{c}), \bar{P})$. The martingale property of $\bar{Y}$ as above gives

$$
E\left[Y_{\infty}\right]=Y_{0}=\gamma_{\epsilon}^{-R} \frac{x^{1-R}}{1-R}
$$

Since $Y_{\infty}=\int_{0}^{\infty} e^{-\rho t}\left(\gamma_{\epsilon} \bar{X}_{t}\right)^{1-R} d t$,

$$
E^{\bar{P}}\left[\int_{0}^{\infty} e^{-\rho t} \frac{\left(\bar{c}_{t}\right)^{1-R}}{1-R} d t\right]=\gamma_{\epsilon}^{-R} \frac{x^{1-R}}{1-R} \leq V_{\Sigma}(0, x)
$$

in which the inequality follows from Lemma 3. By the standard minimax inequality: $\sup \inf \cdots \leq \inf \sup \cdots$,

$$
\begin{aligned}
V_{\Sigma}(0, x) & \leq \inf _{P \in \mathcal{P}_{\Sigma}} \sup _{\mathcal{A}^{\mathrm{rob}}(x)} E^{P}\left[\int_{0}^{\infty} e^{-\rho t} \frac{\left(c_{t}\right)^{1-R}}{1-R} d t\right] \\
& \leq \inf _{\mu \in U_{\epsilon}(\Sigma)} \sup _{\mathcal{A}^{\mathrm{rob}}(x)} E^{P^{\mu}}\left[\int_{0}^{\infty} e^{-\rho t} \frac{\left(c_{t}\right)^{1-R}}{1-R} d t\right]
\end{aligned}
$$


where the second infimum is made on the probabilities corresponding to constant drifts only. Therefore, if we prove that the inf sup value on the right is equal to $\gamma_{\epsilon}^{-R} \frac{x^{1-R}}{1-R}$, we are done. To this end, note that for a fixed constant $\mu \in U_{\epsilon}(\Sigma)$ the inner supremum is a classic Merton problem with parameters $\mu, \Sigma$. Set $\gamma(\mu):=$ $\frac{\rho+(R-1)\left(r+\frac{1}{2} \frac{(H(\mu))^{2}}{R}\right)}{R}$, with Sharpe Ratio $H(\mu):=\sqrt{(\mu-r \mathbf{1})^{\prime} \Sigma^{-1}(\mu-r \mathbf{1})}$. By classic results, when $\gamma(\mu) \leq 0$, such supremum is $\infty$ and when $\gamma(\mu)>0$ (which happens e.g. for $\mu$ close enough to $\bar{\mu}$ ), the supremum is attained and

$$
\max _{(\theta, c) \in \mathcal{A}^{\mathrm{rob}}(x)} E^{\mu}\left[\int_{0}^{\infty} e^{-\rho t} \frac{\left(c_{t}\right)^{1-R}}{1-R} d t\right]=\gamma(\mu)^{-R} \frac{x^{1-R}}{1-R}<\infty
$$

The residual minimization:

$$
\inf _{\mu \in U_{\epsilon}(\Sigma)} \gamma(\mu)^{-R} \frac{x^{1-R}}{1-R}
$$

is a simple exercise, the minimizer being $\bar{\mu}$, which then realizes also the minimal market Sharpe Ratio. So, the minimal $\gamma$ is $\gamma(\bar{\mu})=\gamma_{\epsilon}$, the inequality chain becomes an equality and $\left(\left(\pi_{\epsilon} \bar{X}, \gamma_{\epsilon} \bar{X}\right), P^{\bar{\mu}}\right)$ is a saddle point.

2. High ambiguity case $H_{\epsilon} \leq 0$, or equivalently $r \mathbf{1} \in U_{\epsilon}(\Sigma)$. The candidate $\pi_{\epsilon}=0$, and $\gamma_{\epsilon}=\frac{\rho+(R-1) r}{R}$. The candidate optimal wealth is the deterministic process $\bar{X}_{t}=$ $x \exp \left(\left(r-\gamma_{\epsilon}\right) t\right)$. The process $\bar{Y}$ is constant and equals $\gamma_{\epsilon}^{-R} \frac{x^{1-R}}{1-R}$ so that

$$
\gamma_{\epsilon}^{-R} \frac{x^{1-R}}{1-R} \leq V_{\Sigma}(0, x) \leq \inf _{\mu \in U_{\epsilon}(\Sigma)} \sup _{\mathcal{A}^{\mathrm{rob}}(x)} E^{P^{\mu}}\left[\int_{0}^{\infty} e^{-\rho t} \frac{\left(c_{t}\right)^{1-R}}{1-R} d t\right]
$$

Set $\bar{\mu}:=r \mathbf{1}$, which here is considered a plausible drift. Such a choice for the parameter leads to a classic Merton problem with zero market Sharpe Ratio, whence

$$
\begin{aligned}
V_{\Sigma}(0, x) & \leq \inf _{\mu \in U_{\epsilon}(\Sigma)} \sup _{\mathcal{A}^{\mathrm{rob}}(x)} E^{P^{\mu}}\left[\int_{0}^{\infty} e^{-\rho t} \frac{\left(c_{t}\right)^{1-R}}{1-R} d t\right] \\
& \leq \gamma(\bar{\mu})^{-R} \frac{x^{1-R}}{1-R}=\left(\gamma_{\epsilon}\right)^{-R} \frac{x^{1-R}}{1-R}
\end{aligned}
$$

which shows that all the inequalities are equalities and that $\left(\left(0, \gamma_{\epsilon} \bar{X}\right), P^{r \mathbf{1}}\right)$ is a saddle point.

Remark 4 The reader may wonder why one has to treat the low and high ambiguity cases separately in the verification. Lemma 3 holds also for $\pi=0$, but only as far as the primal value is concerned. When the optimal $\pi_{\epsilon}=0$, the candidate $\bar{X}$ is deterministic, so the expectation does not depend on $P$ anymore. In this case, Lemma 3 simply picks one probability, $P^{\hat{\mu}}$, which needs not correspond to a saddle point.

The result for the logarithmic case has a similar proof, which is then omitted.

Theorem 3 (Logarithmic case) The infinite-horizon robust Merton problem, with logarithmic utility and initial endowment $x>0$, under ellipsoidal ambiguity of mean returns:

$$
V_{\Sigma}(0, x)=\sup _{(\theta, c) \in \mathcal{A}^{\mathrm{rob}}(x)} \inf _{P \in \mathcal{P}_{\Sigma}} E^{P}\left[\int_{0}^{\infty} e^{-\rho t} \ln c_{t} d t\right]
$$


has value function

$$
V_{\Sigma}(0, x)=\frac{\ln x}{\rho}+k_{\epsilon},
$$

in which

$$
k_{\epsilon}=\frac{1}{\rho^{2}}\left[\rho \ln \rho+r-\rho+\frac{\left(H_{\epsilon}^{+}\right)^{2}}{2}\right] .
$$

- The optimal controls are

$$
\bar{\theta}_{t}=\pi_{\epsilon} \bar{X}_{t}, \quad \bar{c}_{t}=\rho \bar{X}_{t},
$$

with optimal portfolio proportions vector given by

$$
\pi_{\epsilon}:=\frac{H_{\epsilon}^{+}}{H} \Sigma^{-1}(\hat{\mu}-r \mathbf{1}) .
$$

The optimal portfolio $\pi_{\epsilon}$ is therefore null if and only if $H_{\epsilon} \leq 0$ or, equivalently, $r \mathbf{1}$ is a plausible drift.

- The optimal adverse control is the probability $\bar{P}$ under which $S$ evolves with constant instantaneous covariance $\Sigma$ and drift

$$
\bar{\mu}:= \begin{cases}\hat{\mu}-\epsilon \frac{\Sigma}{\sqrt{\pi_{\epsilon}^{\prime} \Sigma \pi_{\epsilon}}} \pi_{\epsilon} & \text { if } r \mathbf{1} \notin U_{\epsilon}(\Sigma) \\ r \mathbf{1} & \text { otherwise }\end{cases}
$$

- The optimal drift $\bar{\mu}$ pointwisely realizes the worst market Sharpe Ratio among the plausible models $\mathcal{P}_{\Sigma}$ :

$$
\min _{P \in \mathcal{P}_{\Sigma}} \sqrt{\left(\mu_{t}-r \mathbf{1}\right)^{\prime} \Sigma^{-1}\left(\mu_{t}-r \mathbf{1}\right)}=\text { const }=H_{\epsilon}^{+}
$$

- The optimal wealth process has $\bar{P}$ dynamics given by

$$
\bar{X}_{t}=x \exp \left(\pi_{\epsilon} \sigma W_{t}^{\bar{P}}+\left(r+\left(H_{\epsilon}^{+}\right)^{2}-\rho\right) t\right) .
$$

when $\pi_{\epsilon} \neq 0$, and is the deterministic $\bar{X}_{t}=x \exp ((r-\rho) t)$ otherwise.

- The minimax equality holds:

$$
V_{\Sigma}(0, x)=\inf _{P \in \mathcal{P}_{\Sigma}} \sup _{(\theta, c) \in \mathcal{A}^{\mathrm{rob}}(x)} E^{P}\left[\int_{0}^{\infty} e^{-\rho t} \ln c_{t} d t\right]
$$

and $((\bar{\theta}, \bar{c}), \bar{P})$ is a saddle point for the agent-market game.

Some comments on the above results are in order.

- The optimal portfolio $\bar{\theta}$ preserves the form of the Merton's Mutual Fund theorem. Independently of the agent's utility in the CRRA class, the optimal portfolio consists of an allocation between two fixed mutual funds, namely the riskless asset and the fund of risky assets given by $\Sigma^{-1}(\hat{\mu}-r \mathbf{1})$.

- The optimal wealth proportions vector $\pi_{\epsilon}$ is now dependent on the ambiguity aversion of the investor in addition to his/her risk aversion through the coefficient:

$$
\frac{H_{\epsilon}^{+}}{R H}, \quad R>0
$$

in which $H_{\epsilon}^{+}$is the minimal market Sharpe Ratio among all models, and $H$ is the Sharpe Ratio in the supposed-true one. 
- The optimal $\pi_{\epsilon}$ collapses to the Merton allocation for $\epsilon=0$.

- In case the radius of ambiguity $\epsilon$ is too high, to the extent that the ellipsoid includes the drift $r \mathbf{1}$, naturally the optimal equity demand is 0 .

- Since $\frac{H_{\epsilon}^{+}}{R H} \leq \frac{1}{R}$, the robust Merton portfolio $\pi_{\epsilon}$ has smaller positions in absolute value with respect to the classical Merton portfolio. To wit, both long and short positions are shrunk with respect to the ambiguity-neutral portfolio. As expected, and already anticipated in the Introduction, robustness in the decisions lowers the optimal demand on equity, and thus offers a theoretical basis for a possible explanation of the equity premium puzzle.

\subsection{The finite horizon planning for non ambiguous $\Sigma$}

Assme that the investor has a power utility both from intertemporal and terminal consumption at time $T<\infty$ :

$$
u(t, x)=\mathrm{e}^{-\rho t} \frac{x^{1-R}}{1-R} \quad \text { for } 0 \leq t<T \quad \text { and } \quad u(T, x)=A \frac{x^{1-R}}{1-R}
$$

in which $A$ is a fixed positive constant. Here, we set the deterministic scaling of the CRRA power utility identical to that of the infinite horizon case to better highlight the similarities, but the following results hold also if $e^{-\rho t}$ is replaced by an integrable, positive and deterministic function $h(t)$. The problem is now:

$$
V_{\Sigma}(0, x)=\sup _{(\theta, c) \in \mathcal{A}^{\mathrm{rob}}(x)} \inf _{P \in \mathcal{P}_{\Sigma}} E^{P}\left[\int_{0}^{T} \mathrm{e}^{-\rho s} \frac{c_{s}^{1-R}}{1-R} d s+A \frac{X_{T}^{1-R}}{1-R}\right],
$$

Using the scaling properties of the CRRA utility, the guess to the value function is of the form $v(t, x)=f(t) \frac{x^{1-R}}{1-R}$ for some positive, differentiable function satisfying $f(T)=A$. The HJBI equation (12) now looks like

$$
\begin{aligned}
& \max _{(\theta, c) \in \mathbb{R}^{n} \times \mathbb{R}^{+}}\left[e^{-\rho t} \frac{c^{1-R}}{1-R}+f^{\prime}(t) \frac{x^{1-R}}{1-R}+f(t) x^{-R}\left(r x+\theta^{\prime}(\hat{\mu}-r \mathbf{1})-\epsilon \sqrt{\theta^{\prime} \Sigma \theta}-c\right)\right. \\
& \left.-\frac{R}{2} f(t) x^{-R-1} \theta^{\prime} \Sigma \theta\right]=0 .
\end{aligned}
$$

Proceeding exactly as in the previous section, one obtains

$$
\bar{c}(t, x)=x\left(\frac{e^{-\rho t}}{f(t)}\right)^{1 / R} \bar{\theta}=x \pi_{\epsilon} .
$$

Substituting the above back into the HJBI equation results in a first order ODE for $f$ :

$$
\left\{\begin{array}{l}
f^{\prime}(t)+k_{\epsilon} f(t)+R e^{-\frac{\rho}{R} t}(f(t))^{1-\frac{1}{R}}=0 \\
f(T)=A
\end{array}\right.
$$

with

$$
k_{\epsilon}:=(1-R)\left(r+\pi_{\epsilon}^{\prime}(\hat{\mu}-r \mathbf{1})-\epsilon \sqrt{\pi_{\epsilon}^{\prime} \Sigma \pi_{\epsilon}}-\frac{R}{2} \pi_{\epsilon}^{\prime} \Sigma \pi_{\epsilon}\right)=(1-R)\left(r+\frac{\left(H_{\epsilon}^{+}\right)^{2}}{2 R}\right) .
$$

With the substitution $f(t)=g(t)^{R}$, the ODE can be linearized and easily solved:

$$
g(t)=A^{\frac{1}{R}} \exp \left(\frac{k_{\epsilon}}{R}(T-t)\right)+e^{-\frac{k_{\epsilon}}{R} t} \int_{t}^{T} \exp \left(\frac{k_{\epsilon}-\rho}{R} s\right) d s .
$$


Comparing this to the solution of [27, Section 2.1] the only changes are: 1) the constant $k_{\epsilon}$, in which $H^{2}$ is replaced by $\left(H_{\epsilon}^{+}\right)^{2}$ and 2) the optimal portfolio allocation, which is identical to the robust allocation case of the previous section. By a verification procedure similar to that of the infinite horizon case, one obtains:

Proposition 2 The finite horizon robust Merton problem with power utility, under ellipsoidal ambiguity of mean returns:

$$
V_{\Sigma}(0, x)=\sup _{(\theta, c) \in \mathcal{A}^{\mathrm{rob}}(x)} \inf _{P \in \mathcal{P}_{\Sigma}} E^{P}\left[\int_{0}^{T} e^{-\rho s} \frac{c_{s}^{1-R}}{1-R} d s+A \frac{X_{T}^{1-R}}{1-R}\right],
$$

is finite valued, and admits the optimal controls:

$$
\begin{aligned}
& \bar{\theta}_{t}=\bar{X}_{t} \frac{H_{\epsilon}^{+}}{R H} \Sigma^{-1}(\hat{\mu}-r \mathbf{1})=\bar{X}_{t} \pi_{\epsilon} \\
& \bar{c}_{t}=\bar{X}_{t} \frac{e^{-\frac{\rho}{R} t}}{g(t)}
\end{aligned}
$$

where

$$
g(t)=A^{\frac{1}{R}} \exp \left(\frac{k_{\epsilon}}{R}(T-t)\right)+e^{-\frac{k_{\epsilon}}{R} t} \int_{t}^{T} \exp \left(\frac{k_{\epsilon}-\rho}{R} s\right) d s,
$$

and $k_{\epsilon}=(1-R)\left(r+\frac{\left(H_{\epsilon}^{+}\right)^{2}}{2 R}\right)$. The optimal $\bar{\mu}$ is the same as in the infinite horizon case. The optimal wealth process $\bar{X}$ has dynamics under $\bar{P}:=P^{\bar{\mu}}$ given by:

$$
\bar{X}_{t}=x \exp \left[\left(r+\frac{\left(H_{\epsilon}^{+}\right)^{2}}{2 R^{2}}(R-1)\right) t+\int_{0}^{t} \frac{e^{-\frac{\rho}{R} s}}{g(s)} d s+\pi_{\epsilon}^{\prime} \sigma W_{t}^{\bar{P}}\right] .
$$

Remark 5 The logarithmic problem with finite horizon can be treated in a similar way.

\section{Ambiguity on $\Sigma$ taken into account}

Building on the findings in the constant volatility setup, we now prove general robust case, with $\mathcal{P}$ as in Definition 1.

Theorem 4 Let

$$
\bar{K}:=\operatorname{argmin}_{\Sigma \in K}\left((\hat{\mu}-r \mathbf{1})^{\prime} \Sigma^{-1}(\hat{\mu}-r \mathbf{1})\right)
$$

and $\bar{H}$ denote the square root of the minimum above, namely the Sharpe Ratio

$$
\bar{H}:=\sqrt{(\hat{\mu}-r \mathbf{1})^{\prime} \bar{\Sigma}^{-1}(\hat{\mu}-r \mathbf{1})}, \text { for any } \bar{\Sigma} \in \bar{K}
$$

Let also $\bar{H}_{\epsilon}^{+}=\max (\bar{H}-\epsilon, 0)$. Then:

- Power case The infinite-horizon robust Merton problem, with power utility and initial endowment $x>0$ :

$$
V(0, x)=\sup _{(\theta, c) \in \mathcal{A}^{\mathrm{rob}}(x)} \inf _{P \in \mathcal{P}} E^{P}\left[\int_{0}^{\infty} e^{-\rho t} \frac{c_{t}^{1-R}}{1-R} d t\right],
$$


is finite valued if and only if

$$
\bar{\gamma}_{\epsilon}=\frac{\rho+(R-1)\left(r+\frac{1}{2} \frac{\left(\bar{H}_{\epsilon}^{+}\right)^{2}}{R}\right)}{R}>0
$$

In case $\bar{\gamma}_{\epsilon}>0$, the same conclusions as in Theorem 2 hold with $\gamma_{\epsilon}$ replaced by $\bar{\gamma}_{\epsilon}>0$, and $\Sigma$ replaced by $\bar{\Sigma}$. In particular, the optimal controls are:

1. $\bar{\theta}_{t}=\bar{\pi}_{\epsilon} \bar{X}_{t}, \bar{c}_{t}=\bar{\gamma}_{\epsilon} \bar{X}_{t}$, with optimal portfolio proportions vector given by

$$
\bar{\pi}_{\epsilon}:=\frac{\bar{H}_{\epsilon}^{+}}{R \bar{H}} \bar{\Sigma}^{-1}(\hat{\mu}-r \mathbf{1}) .
$$

2. Any $\bar{P}$ under which $S$ evolves with constant instantaneous covariance $\bar{\Sigma} \in \bar{K}$ and drift

$$
\bar{\mu}:= \begin{cases}\hat{\mu}-\epsilon \frac{\bar{\Sigma}}{\sqrt{\bar{\pi}_{\epsilon}^{\prime} \bar{\Sigma} \bar{\pi}_{\epsilon}}} \bar{\pi}_{\epsilon} & \text { if } r \mathbf{1} \notin U_{\epsilon}(\bar{\Sigma}) \\ r \mathbf{1} & \text { otherwise }\end{cases}
$$

- Log case The infinite-horizon robust Merton problem, with logarithmic utility and initial endowment $x>0$,

$$
V(0, x)=\sup _{(\theta, c) \in \mathcal{A}^{\mathrm{rob}}(x)} \inf _{P \in \mathcal{P}} E^{P}\left[\int_{0}^{\infty} e^{-\rho t} \ln c_{t} d t\right]
$$

is finite valued. The same conclusions as in Theorem 3 hold with $\Sigma$ replaced by $\bar{\Sigma}$, and $k_{\epsilon}$ replaced by

$$
\bar{k}_{\epsilon}=\frac{1}{\rho^{2}}\left[\rho \ln \rho+r-\rho+\frac{\left({\overline{H_{\epsilon}}}^{+}\right)^{2}}{2}\right] .
$$

In particular, the optimal controls are:

1. $\bar{\theta}_{t}=\bar{\pi}_{\epsilon} \bar{X}_{t}, \bar{c}_{t}=\rho \bar{X}_{t}$, with $\bar{\theta}_{t}=\bar{\pi}_{\epsilon} \bar{X}_{t}, \bar{c}_{t}=\bar{\gamma}_{\epsilon} \bar{X}_{t}$, with optimal portfolio proportions vector given by

$$
\bar{\pi}_{\epsilon}:=\frac{\bar{H}_{\epsilon}^{+}}{\bar{H}} \bar{\Sigma}^{-1}(\hat{\mu}-r \mathbf{1}) .
$$

2. Any $\bar{P}$ under which $S$ evolves with instantaneous covariance $\bar{\Sigma} \in \bar{K}$ and drift $\bar{\mu}$ as in the power case.

- Moreover, when $\bar{\gamma}_{\epsilon}>0$, in both the power and logarithmic cases:

1. The equalities

$$
V(0, x)=\inf _{\Sigma \in K} V_{\Sigma}(0, x)=\inf _{P \in \mathcal{P}} \sup _{(\theta, c) \in \mathcal{A}^{\mathrm{rob}}(x)} E^{P}\left[\int_{0}^{\infty} u\left(t, c_{t}\right) d t\right]
$$

hold and $((\bar{\theta}, \bar{c}), \bar{P})$ is a saddle point of the agent-adverse market game;

2. Any optimal couple $(\bar{\Sigma}, \bar{\mu})$ pointwisely realizes the worst Sharpe Ratio among the plausible models $\mathcal{P}$ :

$$
\min _{P \in \mathcal{P}} \sqrt{\left(\mu_{t}-r \mathbf{1}\right)^{\prime} \Sigma_{t}^{-1}\left(\mu_{t}-r \mathbf{1}\right)}=\mathrm{cons} t=\bar{H}_{\epsilon}^{+}
$$


3. The agent does not participate to the stock market $\left(\bar{\pi}_{\epsilon}=0\right)$ if and only if $r \mathbf{1}$ is a plausible drift under a worst case volatility $\bar{\Sigma}$.

Proof We limit the proof to the power case. By Remark 1, any deterministic and bounded proportion plan belongs to $\mathcal{A}^{\mathrm{rob}}(x)$. Specifically, if $\theta_{t}=X_{t} \pi$ and $c_{t}=\lambda_{t} X_{t}$ denotes a plan with constant $\pi \neq 0$ and $\lambda_{t}$ deterministic, under a fixed $P \in \mathcal{P}$ the expected $P$-utility from this plan is:

$$
E^{P}\left[\int_{0}^{\infty} e^{-\rho t} \frac{\left(X_{t} \lambda\right)^{1-R}}{1-R} d t\right]=\int_{0}^{\infty} e^{-\rho t} \frac{\lambda^{1-R}}{1-R} E^{P}\left[X_{t}^{1-R}\right] d t .
$$

If $\left(\Sigma_{t}\right)_{t}$ and $\left(\mu_{t}\right)_{t}$ are the variance-covariance and drift processes under $P, E^{P}\left[X_{t}^{1-R}\right]$ equals

$$
E^{P}\left[\frac{x^{1-R}}{1-R} \exp \left\{(1-R)\left(\int_{0}^{t}\left\{\pi^{\prime}\left(\mu_{s}-r \mathbf{1}\right)-\lambda-\frac{R}{2} \pi^{\prime} \Sigma_{s} \pi\right\} d s\right)\right\}\right] .
$$

Let $\Sigma(\theta)=\Sigma(\pi)$ be an arbitrary element of $\operatorname{argmax}_{K}\left(\pi^{\prime} \Sigma \pi\right)$. The random variable inside the expectation is a monotone increasing function of the integral in the exponent. The proof of Proposition 1 shows that this exponent is minimized pointwisely by the constants $\Sigma(\pi)$ and $\mu(\pi)=\hat{\mu}-\epsilon \frac{\Sigma(\pi) \pi}{\sqrt{\pi^{\prime} \Sigma(\pi) \pi}}$. There exists a unique $P^{\pi, \Sigma(\pi)}$ on the Wiener space such that $S$ evolves with these constant coefficients (see Remark 2). Thus, $P^{\pi, \Sigma(\pi)}$ is $a$ minimizer in $\mathcal{P}$ of the inner problem given the constant plan, and all the minimizers are obtained by letting $\Sigma(\pi)$ vary in the set $\operatorname{argmax}_{K}\left(\pi^{\prime} \Sigma \pi\right)$.

- Low ambiguity case $r \mathbf{1} \notin U_{\epsilon}(\bar{\Sigma})$. The candidate investment proportion $\bar{\pi}_{\epsilon} \neq 0$ and the corresponding $\Sigma\left(\bar{\pi}_{\epsilon}\right)$ s are precisely all the elements of $\bar{K}$. Fix a $\bar{\Sigma}$ in $\bar{K}$ and denote by $V_{\bar{\Sigma}}(0, x)$ the value function of a Merton problem with constant $\bar{\Sigma}$ and ellipsoidal uncertainty on the drift, as examined in the previous Section. Theorem 2, the above results and standard minimax inequalities lead to the next chain

$$
\begin{aligned}
V_{\bar{\Sigma}}(0, x) & =\sup _{\lambda_{t}>0} E^{P^{\bar{\pi}_{\epsilon}, \bar{\Sigma}}}\left[\int_{0}^{\infty} e^{-\rho t} \frac{\left(X_{t} \lambda_{t}\right)^{1-R}}{1-R} d t\right] \\
& \leq \sup _{\pi \in \mathbb{R}^{n}, \lambda_{t}>0} E^{P^{\pi, \Sigma(\pi)}}\left[\int_{0}^{\infty} e^{-\rho t} \frac{\left(X_{t} \lambda\right)^{1-R}}{1-R} d t\right] \leq \\
& \leq V(0, x) \leq \inf _{P \in \mathcal{P}} \sup _{(\theta, c) \in \mathcal{A}^{\mathrm{rob}}(x)} E^{P}\left[\int_{0}^{\infty} e^{-\rho t} \frac{\left(c_{t}\right)^{1-R}}{1-R} d t\right] \leq V_{\bar{\Sigma}}(0, x)
\end{aligned}
$$

Therefore:

1. The inequalities are a fortiori equalities, in particular we get the minimax equality

$$
V(0, x)=\inf _{P \in \mathcal{P}} \sup _{(\theta, c) \in \mathcal{A}^{\mathrm{rob}}(x)} E^{P}\left[\int_{0}^{\infty} e^{-\rho t} \frac{\left(c_{t}\right)^{1-R}}{1-R} d t\right]
$$

and $V(0, x)=\inf _{\Sigma \in K} V_{\Sigma}(0, x)$.

2. The robust Merton problem is finite valued if and only if $V_{\bar{\Sigma}}(0, x)$ is, which by Theorem 2 is equivalent to

$$
\bar{\gamma}_{\epsilon}=\frac{\rho+(R-1)\left(r+\frac{1}{2} \frac{\left(\bar{H}_{\epsilon}^{+}\right)^{2}}{R}\right)}{R}>0 .
$$


3. When $\bar{\gamma}_{\epsilon}>0$, the optimal controls are as stated, with $\bar{P}:=P^{\bar{\pi}_{\epsilon}, \bar{\Sigma}}$, as they realize $V_{\bar{\Sigma}}(0, x)$. Minimality of the Sharpe Ratio $\bar{H}_{\epsilon}^{+}$easily follows.

- High ambiguity case $r \mathbf{1} \in U_{\epsilon}(\bar{\Sigma})$. The candidates are $\bar{\pi}_{\epsilon}=0$, and $\bar{\gamma}_{\epsilon}=\frac{\rho+(R-1) r}{R}$. The candidate optimal wealth is the deterministic process $\bar{X}_{t}=x \exp \left(\left(r-\gamma_{\epsilon}\right) t\right)$. Proceeding as in the last part of Theorem 2, it is straightforward to verify that the candidate controls are indeed optimal, together with any $\bar{P}$ under which $S$ evolves with drift $r \mathbf{1}$ and covariance $\bar{\Sigma} \in \bar{K}$.

As a consequence, the general robust problem becomes observationally equivalent to a CRRA utility maximization with $\bar{P}$ under which $S$ evolves with the worst instantaneous Sharpe Ratio. Effectively, the agent behaves as if the true probability were one of these $\bar{P} \mathrm{~s}$, with wealth equation:

$$
d X_{t}=\left(r X_{t}+\theta_{t}^{\prime}\left(\bar{\mu}_{t}-r \mathbf{1}\right)-c_{t}\right) d t+\theta_{t}^{\prime} \bar{\sigma} d W_{t}^{\bar{P}} .
$$

These results are in accordance with the existing literature. Our contribution differs in that we do not require any specific condition on the volatility structure (as [25]), nor convexity of $K$ or compactness of the strategies (as in [20]).

From a computational point of view, one only needs to find the worst cases matrices set $\bar{K}$. This optimization problem can be solved very easily by standard techniques when $K$ is convex, but convexity is not required for our theoretical results to hold. A particularly simple case is when $K$ has a maximal element $\Sigma^{M}$ with respect to the positive ordering of symmetric matrices:

$$
x^{\prime} \Sigma^{M} x \geq x^{\prime} \Sigma x \quad \text { for all } x \in \mathbb{R}^{n}, \Sigma \in K
$$

It is clear that $\Sigma^{M}$ will be a worst case matrix ${ }^{1}$, as it happens in the two examples below.

Example 1 (The uncorrelated case) Suppose that the risky assets returns are (instantaneously) uncorrelated, with estimated $\hat{\sigma}_{i}^{2}$. Further, assume that the ambiguity does not affect correlations, namely the set $K$ is that of diagonal matrices, whose diagonal lies in some product

$$
\left[\underline{\sigma}_{1}^{2}, \bar{\sigma}_{1}^{2}\right] \times \cdots\left[\underline{\sigma}_{n}^{2}, \bar{\sigma}_{n}^{2}\right]
$$

with $\inf _{i} \underline{\sigma}_{i}>0$ and $\underline{\sigma}_{i} \leq \hat{\sigma}_{i} \leq \bar{\sigma}_{i}$. This is exactly the case examined by Lin and Riedel [25], where the problem is treated via a G-Brownian motion technique. With this diagonal uncertainty specification, the set $K$ as a positive ordering maximum, $\bar{\Sigma}=\operatorname{Diag}\left(\bar{\sigma}_{1}^{2}, \ldots, \bar{\sigma}_{n}^{2}\right)$. By Theorem 4, the optimal relative portfolio is

$$
\pi_{\epsilon}:=\frac{\bar{H}_{\epsilon}^{+}}{R \bar{H}} \operatorname{Diag}\left(\frac{1}{\bar{\sigma}_{1}^{2}}, \ldots, \frac{1}{\bar{\sigma}_{n}^{2}}\right)(\hat{\mu}-r \mathbf{1}) .
$$

Example 2 (Upper bound on the quadratic form $\Sigma$ ) This example can be seen as relaxation of the previous one, in the sense that constraints are not imposed separately on each of the eigenvalues of $\Sigma$, nor $\Sigma$ is diagonal. The quadratic form induced by $\Sigma$ is required not to exceed a given threshold $\bar{\lambda}^{2}>0$ on the unit sphere, with $\bar{\lambda} \geq \hat{\lambda}_{M}$, the latter being the maximum eigenvalue of $\hat{\sigma}$. This amounts to imposing the same bound on the maximum eigenvalue of $\Sigma$. Precisely, the ambiguity set of matrices is

$$
K:=\left\{\Sigma \in \mathbb{R}^{n \times n} \mid 0<x^{\prime} \Sigma x \leq \bar{\lambda}^{2}\|x\|^{2} \text { for all } x \in \mathbb{R}^{n}, x \neq 0\right\} .
$$

1 The inverses in fact will satisfy the opposite inequality for every $x$, in particular for $x=\hat{m u}-r \mathbf{1}$ 
This set has a positive ordering maximum, $\bar{\Sigma}=\bar{\lambda} I_{n}$, in which $I_{n}$ is the identity matrix. By Theorem 4 , the optimal relative portfolio is thus

$$
\bar{\pi}_{\epsilon}(\bar{\Sigma})=\frac{\left(\frac{1}{\bar{\lambda}}\|\hat{\mu}-r \mathbf{1}\|-\epsilon\right)^{+}}{\frac{R}{\bar{\lambda}}\|\hat{\mu}-r \mathbf{1}\|} \frac{1}{\bar{\lambda}^{2}}(\hat{\mu}-r \mathbf{1})=\frac{(\|\hat{\mu}-r \mathbf{1}\|-\bar{\lambda} \epsilon)^{+}}{R\|\hat{\mu}-r \mathbf{1}\|} \frac{1}{\bar{\lambda}^{2}}(\hat{\mu}-r \mathbf{1}) .
$$

\section{Conclusions}

Building on a suitable version of the Martingale Principle of optimal control, we obtain a sup-inf PDE of the Hamilton-Jacobi-Bellman-Isaacs type for the robust utility maximization problem. For the class of the CRRA utilities, we find explicit solutions and provide a saddle point analysis of this agent-adverse market game. The findings are in line with the extant literature, in that the investor is observationally equivalent to one who has distorted, worst case, beliefs on drift and volatility (Eq. (22)). If $R$ is relative risk aversion parameter, then the optimal portfolio is given by the constant wealth proportion

$$
\bar{\pi}_{\epsilon}=\frac{\max (\bar{H}-\epsilon, 0)}{R \bar{H}} \bar{\Sigma}^{-1}(\hat{\mu}-r \mathbf{1})
$$

in which $\bar{\Sigma}$ is the constant worst case covariance matrix, $\bar{H}$ is the Sharpe Ratio computed with drift $\hat{\mu}$ and $\bar{\Sigma}$, and $\max (\bar{H}-\epsilon, 0)$ is the worst market Sharpe ratio. When the ambiguity radius is too high, to the extent that $r \mathbf{1} \in U_{\epsilon}(\bar{\Sigma})$, the pessimistic investor naturally refrains from investing in the risky assets and puts all the money in the safe asset.

The form of the optimal $\bar{\pi}_{\epsilon}$ shows also that the Merton's Mutual Fund Theorem continues to hold in our robust framework. Independently of the agent's utility in the CRRA class, the optimal portfolio consists of an allocation in the safe asset and the mutual fund of risky assets $\bar{\Sigma}^{-1}(\hat{\mu}-r \mathbf{1})$.

Acknowledgements We sincerely thank Fausto Gozzi, Paolo Guasoni and Francesco Russo. Part of this research has been conducted while Sara Biagini was visiting the London School of Economics and Political Sciences, and special thanks go to Constantinos Kardaras for a number of precious conversations on the topic.

Conflict of interest The authors declare that they have no conflict of interest

\section{References}

1. Abel, A.: An exploration of the effects of pessimism and doubt on asset returns. J. Econ. Dyn. Control 26, 1075-1092 (2002)

2. Aliprantis, C.D., Border, K.C.: Infinite Dimensional Analysis: A Hitchhiker's Guide. Springer, Berlin (2007)

3. Cecchetti, S., Lam, P., Mark, N.: Asset pricing with distorted beliefs: are equity returns too good to be true? Am. Econ. Rev. 90, 787-805 (2000)

4. Chen, Z., Epstein, L.: Ambiguity, risk and asset returns in continuous time. Econometrica 70(4), 14031443 (2002)

5. Delage, E., Ye, Y.: Distributionally robust optimization under moment uncertainty with application to data-driven problems. Oper. Res. 58(3), 596-612 (2010)

6. DeMiguel, V., Nogales, F.: Portfolio selection with robust estimation. Operat. Res. 57(3), 560-577 (2009)

7. Evans, L.C., Souganidis, P.E.: Differential games and representation formulas for solutions of HamiltonJacobi-Isaacs equations. Indiana Univ. Math. J. 33, 773-797 (1984)

8. Fabozzi, F.J., Kolm, P.N., Pachamanova, D.A., Focardi, S.M.: Robust Portfolio Optimization and Management. Wiley, New York (2007) 
9. Fabozzi, F.J., Huang, D., Zhou, G.: Robust portfolios: contributions from operations research and finance. Ann. Oper. Res. 176, 191-220 (2010)

10. Fabretti, A., Herzel, S., Pınar, M.Ç.: Delegated portfolio management under ambiguity aversion. Oper. Res. Lett. 42(2), 190-195 (2014)

11. Hernandez-Hernandez, D., Schied, A.: Robust utility maximization in a stochastic factor model. Stat. Decis. 24(3), 109-125 (2006)

12. Föllmer, H., Schied, A., Weber, S.: Robust preferences and robust portfolio choice. In Ciarlet, P, Bensoussan, A, Zhang, Q, (eds) Mathematical Modelling and Numerical Methods in Finance. Handbook of Numerical Analysis vol 15, pp. 29-88, (2009)

13. Garlappi, L., Uppal, R., Wang, T.: Portfolio selection with parameter and model uncertainty: a multi-prior approach. Rev. Financ. Stud. 20(1), 41-81 (2007)

14. Goldfarb, D., Iyengar, G.: Robust portfolio selection problems. Math. Oper. Res. 28(1), 138 (2003)

15. Lim, A., Shanthikumar, J.G., Watewai, T.H.: Robust asset allocation with benchmarked objectives. Math. Financ. 21(4), 643-679 (2011)

16. Maccheroni, F., Marinacci, M., Ruffino, D.: Alpha as ambiguity: robust mean-variance portfolio analysis. Econometrica 81, 1075-1113 (2013)

17. Maccheroni, F., Marinacci, M., Rustichini, A.: Ambiguity aversion, robustness, and the variational representation of preferences. Econometrica 74, 1447-1498 (2006)

18. Maenhout, J.P.: Robust portfolio rules and asset pricing. Rev. Financ. Stud 17, 951-983 (2004)

19. Mehra, R., Prescott, E.C.: The equity premium: a puzzle. J. Monet. Econ. 15(2), 145-161 (1985)

20. Neufeld, A., Nutz, M.: Robust utility maximization with Lévy Processes. Math. Financ. (accepted)

21. Nutz, M.: Utility maximization under model uncertainty in discrete time. Math. Financ. 26(2), 252-268 (2016)

22. Owari, K.: Robust utility maximization with unbounded random endowment. Adv. Math. Econ. 14, 147$181(2011)$

23. Pınar, M.Ç., Tütüncü, R.: Robust profit opportunities in risky financial portfolios. Oper Res Lett. 33, 331-340 (2005)

24. Popescu, I.: Robust mean-covariance solutions for stochastic optimization. Oper. Res. 55(1), 98-112 (2005)

25. Lin, Q., Riedel, F.: Optimal consumption and portfolio choice with ambiguity, Working paper, Center for Mathematical Economics, University of Bielefeld, (2014)

26. Revuz, D., Yor, M.: Continuous Martingales and Brownian Motion. Springer, Berlin (1999)

27. Rogers, L.C.G.: Optimal Investment. Springer, Berlin (2013) 